\title{
Use of Ex Vivo Fluorescence Confocal Microscopy for Detection of Tissue Specific Markers
}

\author{
Laura Bertoni*1, Alessandra Pisciotta ${ }^{1}$, Paola Azzoni ${ }^{1}$, Giulia Bertani ${ }^{1}$, Luca Reggiani Bonetti ${ }^{2}$, Stefano Puliatti ${ }^{3}$, \\ Francesca Farnetani $^{1,4}$, Gianluca Carnevale ${ }^{1}$ and Giovanni Pellacani, ${ }^{1,4}$ \\ ${ }^{1}$ Department of Surgery, Medicine, Dentistry and Morphological Sciences with interest in Transplant, Oncology and Regenerative Medicine, Italy \\ ${ }^{2}$ Department of Medical and Surgical Sciences of Children \& Adults, Italy
}

${ }^{3}$ Department of Urology, Italy

${ }^{4}$ Department of Dermatology, Italy

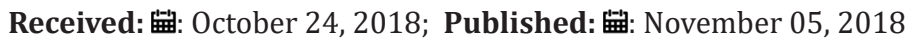

*Corresponding author: Laura Bertoni, Department of Surgery, Medicine, Dentistry and Morphological Sciences with interest in Transplant, Oncology and Regenerative Medicine, Italy

\begin{abstract}
Ex vivo fluorescence confocal microscopy is a novel imaging technique that enables microscopic examination of freshly excised unfixed tissue Prompt examination of the biopsy specimens can be carried out in the operating room, thus offering an alternative to the conventional and timeconsuming histopathological analyses. Ex vivo FCM has been widely investigated in dermatological surgery, including melanoma, basal cell carcinoma, squamous cell carcinoma, as well as cutaneous inflammatory diseases. In this study we aimed to evaluate whether ex vivo FCM Vivascope $2500 \AA$ can provide immunofluorescent images of specific markers through the entire examined specimen formerly labeled by fluorochrome-conjugated antibodies. To this regard, contiguous tissue slides from a colon carcinoma specimen of a 72-year old patient, underwent indirect immunofluorescence analysis of Twist, an epithelial-to-mesenchymal transition marker, and FasL, a key molecule involved in inflammation/immunomodulation events. Our findings demonstrate that ex vivo FCM might offer the possibility to combine a quick evaluation of different tissues to the use of specific fluorescent immunolabeling in order to Ex vivo FCM for detection of tissue markers obtain a panoramic view of the entire examined tissue and of the localization of typical diagnostic markers. The next step might be represented by the development of immunofluorescence protocols with faster incubation times aimed to reach a more precise diagnosis in the intraoperative setting.
\end{abstract}

Keywords: Ex Vivo FCM; Immunofluorescence Analysis; Tissue Markers Detection

Abbreviations: FCM: Fluorescence Confocal Microscopy; PBS: Phosphate Buffer Saline; BSA: Bovine Serum Albumin

\section{Introduction}

Ex vivo Fluorescence Confocal Microscopy (FCM) is an emerging tool that allows to obtain real-time images with nuclear-level resolution of fresh tissue excisions, by staining with fluorescent dyes, in order to capture specimen images closely comparable to frozen sections and routine histopathology. Interestingly, these images can be analyzed right away in the operating room and the tissue, being not altered by ex vivo FCM evaluations, can then undergo histopathological and immunohistochemical evaluations [1]. So far, ex vivo FCM has been widely applied to dermatological surgery, including residual basal cell carcinoma and squamous cell carcinoma during Mohs surgery [2-4]. In a previous study we demonstrated that the ex vivo FCM Vivascope 2500® (Lucid Inc; Rochester NY, USA) allows to obtain differential diagnosis of four of the most common skin inflammatory diseases [1]. Due to its technical features it permits to operate under both reflectance and fluorescence modes, the latter one providing the possibility to widen its range of applicability. Indeed, the identification of specific tissue components and markers by fluorochrome-conjugated antibodies would help in reaching a definitive diagnosis without the need for conventional histological and immunohistochemical staining methods. Combining a prompt examination to the use of specific fluorescent immunolabeling would help in obtaining a panoramic view of the entire examined tissue and of the localization of typical diagnostic markers.

\section{Case Presentation}

In this study we took under consideration a colorectal cancer biopsy obtained from a 72-year old patient who was diagnosed and 
treated at the University Hospital Policlinico of Modena, Modena (Italy). The biopsy specimen was first embedded in paraffin, then $5 \mu \mathrm{m}$ thick serial sections were obtained by using a HM 315 Microm microtome and were processed for routine immunofluorescence analysis, as previously described [5]. Briefly, rabbit anti-Twist (Abcam) and rabbit anti-FasL (Santa Cruz Biotechnology) primary antibodies were diluted 1:100 in Phosphate Buffer Saline (PBS) containing 3\% Bovine Serum Albumin (BSA) and incubated for 1 hour at room temperature. Secondary antibodies were diluted 1:200 in PBS containing 3\% BSA (goat anti-rabbit Alexa488; Thermo Fisher Scientific) and incubated for 1 hour at room temperature.

As formerly described by our group [1], the ex vivo FCM Vivascope $2500 \AA$ has 3 lasers, which enable tissue examination in reflectance $(830 \mathrm{~nm})$ and/or fluorescence (488nm and $658 \mathrm{~nm}$ ) modalities. In our study, $488 \mathrm{~nm}$ fluorescence mode only was used for image acquisition. The software VivaScan ${ }^{\circledR}$ (Version 11, Mavig GmbH, Munich, Germany) enabled the reconstruction of the images from the probes, with the VivaBlock ${ }^{\circledR}$ tool (acquisition of multiple images in the $\mathrm{X} / \mathrm{Y}$ directions within a single plane at a fixed depth) and the VivaStack $®$ tool (permits a survey of multiple frames along the $\mathrm{Z}$ axis, visualizing deeper tissue). The imaging process took a few minutes. Histomorphology of normal mucosa close to colon cancer is shown in Figure 1A. On the right side a wide area of normal mucosa adjacent to colon carcinoma revealed a clear staining against the epithelial-to mesenchymal transition marker Twist. As reported in Figure 1B, areas of colon carcinoma were characterized by a clear inflammatory infiltrate as revealed by Hematoxylin \& Eosin. The fluorescence labelling against FasL revealed a positive staining of inflammatory cells and the absence of non-specific background (Figure 1B, right side).

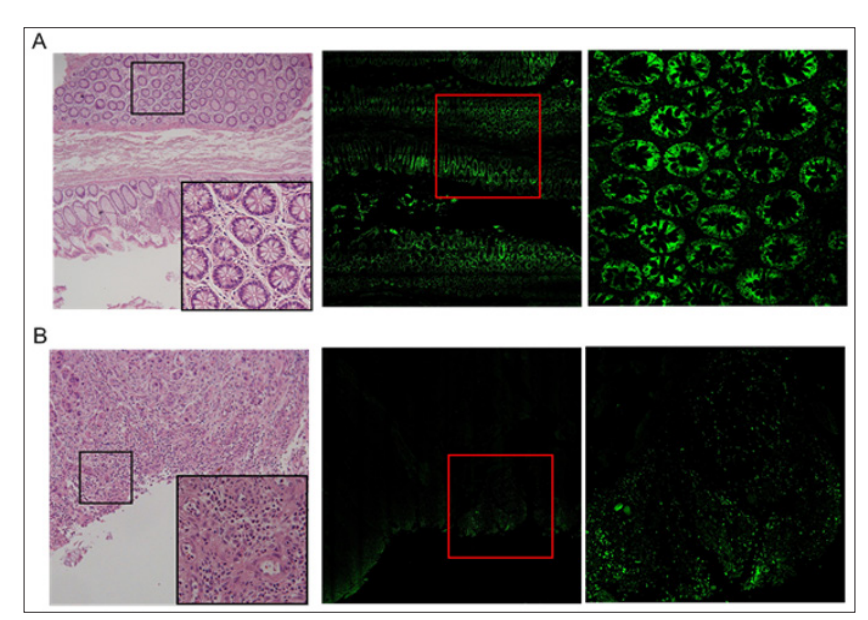

Figure 1: A) On the left, histological image of the specimen, on the right immunofluorescence staining against Twist, showing mucosa close to colon carcinoma. The red square refers to a histological detail, reported on the right as a digital magnification. B) On the left, histological image of the specimen, on the right immunofluorescence staining against FasL, expressed by inflammatory infiltrate in colon carcinoma. The red square refers to a histological detail, reported on the right as a digital magnification.

\section{Discussion}

When using ex vivo FCM, the labelling with acridine orange permits to create high contrast between cellular and cytoplasmic components of the tissues, thus reflecting the specimen architecture [4]. The tissue samples can be evaluated either through reflectance and fluorescence modes operated by ex vivo FCM, in order to promptly provide histomorphology details. In our study paraffin embedded sections were used to evaluate whether the ex vivo FCM Vivascope $2500 \AA$ can provide immunofluorescent images of specific markers on the entire examined specimen formerly labeled by fluorochrome-conjugated antibodies. In particular, here we detected with high specificity the presence of areas expressing an epithelial-to-mesenchymal transition marker and confirmed the presence of inflammatory infiltrate by FasL staining. The immunofluorescence analysis on freshly excised tissues would, of course, require faster incubation times, to be used in real-time intraoperative settings. Experimental measures concerning the thickness of the specimen, the choice of specific fluorochromeconjugated primary antibodies as well as their optimal working dilutions must be considered for the development of an effective, standardized protocol that permits to use ex vivo FCM for faster and more detailed tissue examinations in the intraoperative setting.

Conflict of interest: The authors declare that no conflicts of interest exist.

\section{References}

1. Bertoni L, Azzoni P, Reggiani C, Pisciotta A, Carnevale G, et al. (2018) Ex vivo fluorescence confocal microscopy for intraoperative, real-time diagnosis of cutaneous inflammatory diseases: A preliminary study. Exp Dermatol 27(10): 1152-1159.

2. Longo C, Borsari S, Pampena R, Benati E, Bombonato C, et al. (2018) Basal cell carcinoma: The utility of in vivo and ex vivo confocal microscopy. J Eur Acad Dermatol Venereol [Epub ahead of print].

3. Longo C, Ragazzi M, Gardini S, Piana S, Moscarella E, et al. (2015) Ex vivo fluorescence confocal microscopy in conjunction with Mohs 
micrographic surgery for cutaneous squamous cell carcinoma. J Am Acad Dermatol 73(2): 321-322.

4. Hartmann D, Krammer S, Bachmann MR, Mathemeier L, Ruzicka T, et al (2018) Ex vivo confocal microscopy features of cutaneous squamous cell carcinoma. J Biophotonics 11(4): e201700318.

\section{ISSN: 2574-1241}

DOI: $10.26717 / B J S T R .2018 .10 .002003$

Laura Bertoni. Biomed J Sci \& Tech Res

(c) (P) This work is licensed under Creative

Submission Link: https://biomedres.us/submit-manuscript.php
5. Carnevale G, Pisciotta A, Riccio M, Bertoni L, De Biasi S, et al. (2018) Human dental pulp stem cells expressing STRO-1, c-kit and CD34 markers in peripheral nerve regeneration. J Tissue Eng Regen Med 12(2): e774-e785.

$\begin{array}{ll}\text { BIOMEDICAL } & \text { Assets of Publishing with us } \\ \text { RESEARCHES } & \text { - Global archiving of articles } \\ & \text { - Immediate, unrestricted online access } \\ & \text { - Rigorous Peer Review Process } \\ \end{array}$

